\title{
FACTORING COMPACT AND WEAKLY COMPACT OPERATORS THROUGH REFLEXIVE BANACH LATTICES ${ }^{1}$
}

BY

\author{
C. D. ALIPRANTIS AND O. BURKINSHAW
}

\begin{abstract}
When does a weakly compact operator between two Banach spaces factor through a reflexive Banach lattice?

This paper provides some answers to this question. One of the main results: If an operator between two Banach spaces factors through a Banach lattice with weakly compact factors, then it also factors through a reflexive Banach lattice. In particular, the square of a weakly compact operator on a Banach lattice factors through a reflexive Banach lattice.

Similar results hold for compact operators. For instance, the square of a compact operator on a Banach lattice factors with compact factors through a reflexive Banach lattice.
\end{abstract}

1. Preliminaries. For terminology concerning Riesz spaces and Banach lattices, we follow [2 and 17]. In this section, we briefly review a few basic results concerning Banach lattices.

Let $X$ be a Riesz space. Then the symbol $X^{+}$will denote the positive cone of $X$, i.e., $X^{+}=\{x \in X: x \geqslant 0\}$. The sets of the form

$$
[-x, x]:=\{y \in X:-x \leqslant y \leqslant x\}, \quad x \in X^{+},
$$

are called the order intervals of $X$. A subset $A$ of $X$ is said to be solid whenever $|x| \leqslant|y|$ in $X$ and $y \in A$ imply $x \in A$. The solid hull of a set $A$ is the smallest solid set that contains $A$ and is precisely the $\operatorname{set} \operatorname{sol}(A):=\{x \in X: \exists y \in A$ with $|x| \leqslant|y|\}$. A solid vector subspace of $X$ is referred to as an ideal of $X$.

In this paper the word "operator" is synonymous with "linear operator." An operator $T: X \rightarrow Z$ between two Riesz spaces is said to be positive, in symbols $0 \leqslant T$, whenever $x \in X^{+}$implies $T(x) \in Z^{+}$.

A norm $\|\cdot\|$ on a Riesz space is said to to be a lattice norm whenever $|x| \leqslant|y|$ implies $\|x\| \leqslant\|y\|$. A Banach lattice is a Riesz space equipped with a lattice norm under which it is a Banach space. A Banach lattice is said to have order continuous norm whenever $x_{\alpha} \downarrow 0$ implies $\left\|x_{\alpha}\right\| \downarrow 0$. The closed unit ball of an arbitrary Banach space $Z$ will be denoted by $B_{Z}$; i.e., $B_{Z}:=\{z \in Z:\|z\| \leqslant 1\}$.

Received by the editors April 18, 1983 and, in revised form, September 15, 1983. Presented to the Society, January $27,1984$.

1980 Mathematics Subject Classification. Primary 47A68, 47B55, 46B30, 46A40.

Key words and phrases. Weakly compact operators, factoring operators, reflexive Banach lattices.

${ }^{1}$ Research supported in part by NSF grants MCS 81-00787 and MCS 82-19750.

1984 American Mathematical Society $0025-5726 / 84 \$ 1.00+\$ .25$ per page 
For our work, we shall need the following characterization of Banach lattices with order continuous norms. (We shall denote the norm dual of an arbitrary Banach space $Z$ by $Z^{\prime}$ and its second dual by $Z^{\prime \prime}$.)

THEOREM 1.1. For a Banach lattice $X$ the following statements are equivalent:

1. X has order continuous norm.

2. Each order interval of $X$ is weakly compact.

3. $X$ is an ideal of $X^{\prime \prime}$.

4. Every relatively weakly compact subset of $X^{+}$has a relatively weakly compact solid hull.

Proof. For the equivalence of (1), (2), and (3) see [17, Theorem 5.10, p. 89].

(3) $\Rightarrow$ (4) Let $A \subseteq X^{+}$be relatively weakly compact, and let $x^{\prime \prime} \in X^{\prime \prime}$ be a $w^{*}$-closure point of $\operatorname{sol}(A)$. Pick a net $\left\{x_{\alpha}\right\} \subseteq \operatorname{sol}(A)$ with $x_{\alpha} \stackrel{n^{* *}}{\rightarrow} x^{\prime \prime}$. For each $\alpha$, choose some $y_{\alpha} \in A$ with $-y_{\alpha} \leqslant x_{\alpha} \leqslant y_{\alpha}$. By passing to a subnet, we can assume that $y_{\alpha} \stackrel{\text { w }}{\rightarrow} y$ holds in $X$. This implies $-y \leqslant x^{\prime \prime} \leqslant y$ in $X^{\prime \prime}$, and so, $x^{\prime \prime} \in X$. Therefore, $\operatorname{sol}(A)$ is relatively weakly compact. (For an alternate proof of this implication see [18, Proposition 2.1, p. 241].)

$(4) \Rightarrow(2)$ Note that for each $x \in X^{+}$we have $\operatorname{sol}\{x\}=[-x, x]$ and that $[-x, x]$ is weakly compact.

It should be noted that in a Banach lattice with order continuous norm the solid hull of a relatively weakly compact set need not be relatively weakly compact $[16, \mathrm{p}$. 308]. However, there are some Banach lattices in which the solid hull of a relatively weakly compact set remains relatively weakly compact. These are the KB-spaces, and they will be needed for our work.

Following the Russian literature, we say that a Banach lattice $X$ is a $K B$-space (Kantorovič-Banach space) whenever every increasing norm bounded sequence of $X^{+}$is norm convergent. Clearly, every KB-space has order continuous norm.

THEOREM 1.2. The norm dual $X^{\prime}$ of a Banach lattice $X$ is a KB-space if and only if $X^{\prime}$ has order continuous norm.

Proof. Assume that $X^{\prime}$ has order continuous norm. Let $0 \leqslant x_{n}^{\prime} \uparrow$ hold in $X^{\prime}$ with $\sup \left\{\left\|x_{n}^{\prime}\right\|\right\}<\infty$. Then $x^{\prime}(x)=\lim x_{n}^{\prime}(x)$ exists in $\mathbf{R}$ for each $x \in X$, and moreover, this formula defines a positive linear functional on $X$. Thus, $x^{\prime} \in X^{\prime}$, and clearly, $x_{n}^{\prime} \uparrow x^{\prime}$ holds in $X^{\prime}$. Now note that $\lim \left\|x_{n}^{\prime}-x^{\prime}\right\|=0$.

Recall that if $X$ is a Riesz space, then the symbol $X_{n}^{\sim}$ denotes the order continuous dual of $X$, i.e., the Riesz space of all linear functionals $f$ on $X$ such that $x_{\alpha} \downarrow 0$ in $X$ implies $\lim \left|f\left(x_{\alpha}\right)\right|=0$.

The next result presents some characterizations of KB-spaces. For a proof see [2, p. $159 ; 17$, p. 95 ; or 16, p. 309].

THEOREM 1.3. For a Banach lattice $X$ the following statements are equivalent:

1. $X$ is a KB-space.

2. $X$ is a band of $X^{\prime \prime}$.

3. $X=\left(X^{\prime}\right)_{n}$. 
In a KB-space a relatively weakly compact set has a relatively weakly compact solid hull. This result appeared in [1]; see also [17, Exercise 30, p. 153].

THEOREM 1.4. In a KB-space the convex solid hull of a relatively weakly compact set remains relatively weakly compact.

Proof. Let $A$ be a relatively weakly compact subset of $X$, and consider $A$ as a subset of $\left(X^{\prime}\right)_{n}^{\sim}$. By [2, Corollary 20.12, p. 140] we know that $A$ is relatively $\sigma\left(\left(X^{\prime}\right)_{n}^{\sim}, X^{\prime}\right)$-compact if and only if its convex solid hull is also relatively $\sigma\left(\left(X^{\prime}\right)_{n}^{\sim}, X^{\prime}\right)$-compact. By Theorem 1.3 we have $X=\left(X^{\prime}\right)_{n}^{\sim}$, and our conclusion follows.

For constructing reflexive Banach spaces from weakly compact sets, we shall need the following basic result of [7] (see also [8, p. 250]).

TheOREM 1.5 (DAVIS-FIgIEL-JOHNSON -PEŁCZYŃSKI). Let $X$ be a Banach space with closed unit ball $B=\{x \in X:\|x\| \leqslant 1\}$, and let $W$ be a convex, symmetric, norm bounded subset of $X$. For each $n$ put $U_{n}:=2^{n} W+2^{-n} B$, and denote by $\|\cdot\|_{n}$ the Minkowski functional of $U_{n}$, i.e.,

$$
\|x\|_{n}:=\inf \left\{\alpha>0: x \in \alpha U_{n}\right\} \text {. }
$$

Set $Y=\left\{x \in X:\|x \mid\|=\left(\sum_{n=1}^{\infty}\|x\|_{n}^{2}\right)^{1 / 2}<\infty\right\}$, and let $J: Y \rightarrow X$ denote the natural inclusion. Then:

1. $(Y,||||||)$ is a Banach space and $J$ is continuous;

2. $W$ is a subset of the closed unit ball of $(Y,\||\cdot|\|)$;

3. $J^{\prime \prime}: Y^{\prime \prime} \rightarrow X^{\prime \prime}$ is one-to-one; and

4. $(Y,|\| \cdot|||)$ is reflexive if and only if $W$ is a relatively weakly compact subset of $X$.

Another important property associated with the Banach space $(Y,\|\cdot\| \mid)$, needed for our work, is described in the next lemma.

LEMMA 1.6. Let $W$ be a convex, symmetric, norm bounded subset of a Banach space $X$, and let $(Y, \||\cdot| \mid)$ be the Banach space of Theorem 1.5 determined by $W$. Then a subset of $W$ is totally bounded in $X$ if and only if it is totally bounded in $Y$.

In particular, if a compact operator $T: Z \rightarrow X$ satisfies $T\left(B_{Z}\right) \subseteq W$, then $T$ considered as an operator from $Z$ into $Y$ is also compact.

Proof. Let $D$ be a subset of $W$ which is totally bounded in $X$, and let $0<\varepsilon<1$. Since $x, y \in W$ implies

$$
x-y \in W+W=2 W=2^{1-n}\left(2^{n} W\right) \subseteq 2^{1-n} U_{n},
$$

we see that $\|x-y\|_{n} \leqslant 2^{1-n}$ holds for all $n$, and so, there exists some $k$ satisfying $\sum_{n=k}^{\infty}\|x-y\|_{n}^{2}<\varepsilon$ for all $x, y \in W$. Pick $x_{1}, \ldots, x_{m} \in D$ such that

$$
D \subseteq\left\{x_{1}, \ldots, x_{m}\right\}+\varepsilon 2^{-2 k} B,
$$

where $B$ is the closed unit ball of $X$.

Now let $x \in D$ be fixed. Choose some $x_{i}$ with $\left\|x-x_{i}\right\|<\varepsilon 2^{-2 k}$. Then for $1 \leqslant n \leqslant k$ we have

$$
x-x_{i} \in \varepsilon 2^{-2 k} B \subseteq \varepsilon 2^{-k} 2^{-n} B \subseteq \varepsilon 2^{-k} U_{n},
$$


and so, $\left\|x-x_{i}\right\|_{n} \leqslant \varepsilon 2^{-k}$ holds for all $1 \leqslant n \leqslant k$. Therefore,

$$
\left\|x-x_{i}\right\|^{2} \leqslant \sum_{n=1}^{k}\left\|x-x_{i}\right\|_{n}^{2}+\sum_{n=k}^{\infty}\left\|x-x_{i}\right\|_{n}^{2}<k \varepsilon^{2} 2^{-2 k}+\varepsilon<\varepsilon+\varepsilon=2 \varepsilon
$$

holds, from which it follows that $D$ is also a totally bounded subset of $Y$.

Recall that a positive operator $T: Z \rightarrow X$ between two Riesz spaces is said to be:

(a) interval preserving, whenever $T[0, x]=[0, T x]$ holds for all $x \in Z^{+}$; and

(b) a lattice homomorphism, whenever $T(x \vee y)=T(x) \vee T(y)$ holds for all $x, y \in Z$.

In the sequel, the expression "an operator $T: Z \rightarrow X$ between two Banach spaces is positive" will mean that $Z$ and $X$ are both Banach lattices and that $T$ is a positive operator (i.e., $z \geqslant 0$ implies $T(z) \geqslant 0$ ).

When $X$ is a Banach lattice and $W$ is also a solid set, then the Banach space $(Y,\||\||)$ of Theorem 1.5 is itself a Banach lattice.

THEOREM 1.7. Let $W$ be a convex, solid, and norm bounded subset of a Banach lattice X. If $(Y,\||\|\mid\|)$ is the Banach space of Theorem 1.5 determined by $W$, then we have:

1. $(Y,\||\cdot|\|)$ is a Banach lattice and $Y$ is an ideal of $X$;

2. the operator $J: Y \rightarrow X$ is an interval preserving lattice homomorphism; and

3. $J^{\prime}: X^{\prime} \rightarrow Y^{\prime}$ is also an interval preserving lattice homomorphism.

Proof. (1) Let $|x| \leqslant|y|$ hold in $X$ with $y \in Y$. If $y \in \alpha U_{n}$, then (in view of the solidness of $U_{n}=2^{n} W+2^{-n} B$ ) we see that $x \in \alpha U_{n}$, and so, $\|x\|_{n} \leqslant\|y\|_{n}$. Therefore, $\||x|\| \leqslant\|y\| \|$ holds. This shows that $Y$ is an ideal of $X$, and that $(Y,\|\| \cdot \|)$ is a Banach lattice.

(2) Obvious.

(3) It follows immediately from [15, Proposition 1.2, p. 89].

2. Factoring weakly compact operators. Let $T: Z \rightarrow X$ be a continuous operator between two Banach spaces. Recall that $T$ is said to factor through a Banach space $Z_{1}$ whenever there exist continuous operators $Z \stackrel{S}{\rightarrow} Z_{1} \stackrel{R}{\rightarrow} X$ satisfying $T=R S$. (The operators $R$ and $S$ are called factors of $T$.) By [7] we know that every weakly compact operator factors through a reflexive Banach space. Also, by [10 and 14] we know that every compact operator factors with compact factors through a reflexive Banach space. In this section we show how a weakly compact operator can be factored through a reflexive Banach lattice.

We start our discussion with a theorem that will be the basis for our results.

THEOREM 2.1. Let $W$ be the convex solid hull of a relatively weakly compact subset of a Banach lattice $X$, and let $(Y, \||\cdot| \mid)$ be the Banach lattice as defined in Theorem 1.5. Then $Y^{\prime}$ has order continuous norm (and hence, it is a KB-space).

Proof. By Theorem 1.7, $J^{\prime}: X^{\prime} \rightarrow Y^{\prime}$ is interval preserving, and so, $J^{\prime}\left(X^{\prime}\right)$ is an ideal of $Y^{\prime}$. On the other hand, it is also true that $J^{\prime}\left(X^{\prime}\right)$ is weakly dense in $Y^{\prime}$. Indeed, if some $y^{\prime \prime} \in Y^{\prime \prime}$ satisfies $y^{\prime \prime}\left(J^{\prime} x^{\prime}\right)=0$ for all $x^{\prime} \in X^{\prime}$, then $J^{\prime \prime} y^{\prime \prime}\left(x^{\prime}\right)=0$ 
holds for all $x^{\prime} \in X^{\prime}$, and so, $J^{\prime \prime} y^{\prime \prime}=0$. Since $J^{\prime \prime}: Y^{\prime \prime} \rightarrow X^{\prime \prime}$ is one-to-one, we get $y^{\prime \prime}=0$. Thus, $J^{\prime}\left(X^{\prime}\right)$ is weakly dense in $Y^{\prime}$, and hence, norm dense in $Y^{\prime}$. Now if $J^{\prime}\left(X^{\prime}\right)$ has order continuous norm, then its norm closure $Y^{\prime}$ will also have order continuous norm; see [2, Theorems 17.9 and 10.6]. Therefore, in order to establish that $Y^{\prime}$ has order continuous norm, it is enough to show that $J^{\prime} x_{n}^{\prime} \downarrow 0$ in $J^{\prime}\left(X^{\prime}\right)$ implies ||$J^{\prime} x_{n}^{\prime} \mid \| \downarrow 0$.

To this end, let $J^{\prime} x_{n}^{\prime} \downarrow 0$ in $J^{\prime}\left(X^{\prime}\right)$. Since $J^{\prime} x_{n}^{\prime}=\left|J^{\prime} x_{n}^{\prime}\right|=J^{\prime}\left|x_{n}^{\prime}\right|$ holds, we can assume that $x_{n}^{\prime} \geqslant 0$ for each $n$. Now let $\varepsilon>0$. Fix some $k$ with $2^{-k}\left\|x_{1}^{\prime}\right\|<\varepsilon$. Since $W$ is the convex solid hull of a relatively weakly compact subset of $X$, it follows from [2, Theorems 20.9 and 20.6] that there exists some $u \geqslant 0$ in the ideal generated by $W$ in $X$ satisfying

$$
x_{1}^{\prime}(|w|-u)^{+}<2^{-k} \varepsilon \quad \text { for all } w \in W
$$

From $W \subseteq Y$ and the fact that $Y$ is an ideal of $X$, we see that $u \in Y$. Next choose some $m$ with

$$
2^{k} J^{\prime} x_{n}^{\prime}(u)<\varepsilon \text { for all } n \geqslant m
$$

Now if $y \in Y$ satisfies $\|y\| \leqslant 1$, then $\|y\|_{k} \leqslant 1$ also holds, and so,

$$
y \in 2\left(2^{k} W+2^{-k} B\right)
$$

Write $y=2\left(2^{k} w+2^{-k} v\right)$ with $w \in W$ and $v \in B$. Clearly, $v \in Y$, and moreover,

$$
\begin{aligned}
\left|J^{\prime} x_{n}^{\prime}(y)\right| & \leqslant 2\left[2^{k} J^{\prime} x_{n}^{\prime}(|w|)+2^{-k} J^{\prime} x_{n}^{\prime}(|v|)\right] \\
& \leqslant 2\left[2^{k} J^{\prime} x_{n}^{\prime}(|w|-u)^{+}+2^{k} J^{\prime} x_{n}^{\prime}(u)+2^{-k} x_{n}^{\prime}(|v|)\right] \\
& \leqslant 2\left[2^{k} x_{1}^{\prime}(|w|-u)^{+}+2^{k} J^{\prime} x_{n}^{\prime}(u)+2^{-k} \| x_{1}^{\prime}||\right] \\
& \leqslant 2\left[2^{k} \cdot 2^{-k} \varepsilon+\varepsilon+\varepsilon\right]=6 \varepsilon
\end{aligned}
$$

holds for all $n \geqslant m$. This implies $\left\|\mid J^{\prime} x_{n}^{\prime}\right\| \leqslant 6 \varepsilon$ for all $n \geqslant m$, and hence, $\left\|J^{\prime} x_{n}^{\prime} \mid\right\| \downarrow 0$, as desired.

The next theorem deals with the factorization of a weakly compact operator whose range is in a Banach lattice.

THEOREM 2.2. Let $T: Z \rightarrow X$ be a weakly compact operator from a Banach space $Z$ into a Banach lattice $X$. If the solid hull of $T\left(B_{Z}\right)$ is relatively weakly compact, then $T$ factors through a reflexive Banach lattice.

If, in addition, $T$ is a positive operator, then the factors can be taken to be positive operators.

Proof. Denote by $W$ the convex hull of the solid hull of $T\left(B_{Z}\right)$, i.e., $W=$ $\operatorname{co}\left(\operatorname{sol}\left(T\left(B_{Z}\right)\right)\right)$. By [2, Theorem 1.3, p. 4] the set $W$ is a solid set, and by our hypothesis, it follows that $W$ is also relatively weakly compact. Let $(Y,|\|\cdot\||)$ be the reflexive Banach lattice corresponding to $W$ (Theorems 1.5 and 1.7), and let 
$S: Z \rightarrow Y$ be the operator defined by $S(z)=T(z)$ for all $z \in Z$. Now a glance at the diagram

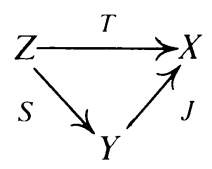

completes the proof of the theorem.

The following is an immediate consequence of the preceding result and Theorem 1.4 .

COROLlary 2.3. Every weakly compact operator $T: Z \rightarrow X$ from a Banach space into a KB-space factors through a reflexive Banach lattice.

Note. Theorem 2.2 and Corollary 2.3 can be derived also from [11, Theorem 3.1, p. 403].

For positive operators the following version holds.

Corollary 2.4. Let $T: Z \rightarrow X$ be a positive weakly compact operator between two Banach lattices. If $X$ has order continuous norm, then $T$ factors (with positive factors) through a reflexive Banach lattice.

Proof. Note that $\operatorname{sol}\left(T\left(B_{Z}\right)\right)=\operatorname{sol}\left(T\left(B_{Z}^{+}\right)\right)$holds. Taking into account Theorem 1.1(4), we see that $T\left(B_{Z}\right)$ has a relatively weakly compact solid hull in $X$. Now apply Theorem 2.2.

The following result describes a factorization property of weakly compact operators whose domains are Banach lattices.

THEOREM 2.5. Let $T: X \rightarrow Z$ be a weakly compact operator between two Banach spaces. If $X$ is a Banach lattice such that $X^{\prime}$ has order continuous norm, then there exist a reflexive Banach lattice $E$ and a factorization of $T$

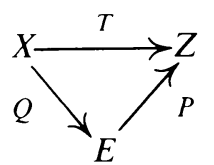

with the factor $Q$ a lattice homomorphism and the factor $P$ positive if $T$ is positive.

Moreover, if another operator $X \stackrel{S}{\rightarrow} Z$ satisfies $0 \leqslant S \leqslant T$, then $S$ admits a factorization through the reflexive Banach lattice $E$

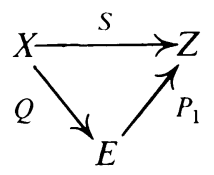

with $0 \leqslant P_{1} \leqslant P$.

Proof. Clearly, $T^{\prime}: Z^{\prime} \rightarrow X^{\prime}$ is weakly compact, and by Theorem 1.2, the Banach lattice $X^{\prime}$ is a $\mathrm{KB}$-space. Thus, by Theorem 1.4 , if $W$ denotes the convex solid hull of $T^{\prime}\left(B_{Z^{\prime}}\right)$, then $W$ is a relatively weakly compact subset of $X^{\prime}$. Let $(Y,\||\|\mid\|)$ be the reflexive Banach lattice of Theorems 1.5 and 1.7 determined by $W$ (where $Y$ is also 
an ideal of $\left.X^{\prime}\right)$. Next, define the operator $R: Z^{\prime} \rightarrow(Y,\|\cdot\| \|)$ by $R\left(z^{\prime}\right)=T^{\prime}\left(z^{\prime}\right)$, and note that $R$ is continuous (and that $R$ is positive if $T$ is positive). Thus, we have the diagrams:

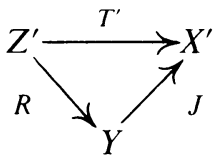

and

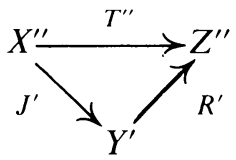

Since $J^{\prime}\left(X^{\prime \prime}\right)$ is dense in $Y^{\prime}$ and $T^{\prime \prime}\left(X^{\prime \prime}\right) \subseteq Z$, we see that $R^{\prime}\left(Y^{\prime}\right) \subseteq Z$. Now consider the reflexive Banach lattice $E=Y^{\prime}$ and the continuous operators $X \stackrel{Q}{\rightarrow} E \stackrel{P}{\rightarrow} Z$ where $P(u)=R^{\prime}(u)$ for $u \in E$ and $Q(x)=J^{\prime}(x)$ for $x \in X$. By Theorem 1.7, $Q$ is a lattice homomorphism, and clearly, $P$ is positive if $T$ is positive. Now note that $T=P Q$ holds.

Now assume $0 \leqslant S \leqslant T$. Then $S^{\prime}\left(Z^{\prime}\right) \subseteq Y$ holds. On the other hand, by [4, Theorem 7] $S$ is also weakly compact, and hence, $S^{\prime \prime}\left(X^{\prime \prime}\right) \subseteq Z$ also holds. As above, this implies that the operator $S_{1}: Z^{\prime} \rightarrow Y$ defined by $S_{1}\left(z^{\prime}\right)=S^{\prime}\left(z^{\prime}\right)$ satisfies $S_{1}^{\prime}\left(Y^{\prime}\right) \subseteq Z$. Define $P_{1}: Y^{\prime} \rightarrow Z$ by $P_{1}\left(y^{\prime}\right)=S_{1}^{\prime}\left(y^{\prime}\right)$, and note that $0 \leqslant P_{1} \leqslant P$ and $S=P_{1} Q$ hold. The proof of the theorem is now complete.

Consider two positive operators $S, T: X \rightarrow X$ on a Banach lattice satisfying $0 \leqslant S \leqslant T$. By [4, Example 1] we know that if $T$ is weakly compact then $S$ need not be weakly compact. However, we proved in [4] that if $T$ is weakly compact, then $S^{2}$ is necessarily weakly compact. The next theorem is a generalization of this result.

THEOREM 2.6. Consider the scheme of weakly compact operators

$$
Z_{1} \stackrel{T_{1}}{\rightarrow} X \stackrel{T_{2}}{\rightarrow} Z_{2}
$$

between Banach spaces. If $X$ is a Banach lattice, then there exist a reflexive Banach lattice $E$ and a factorization of $T_{2} T_{1}$

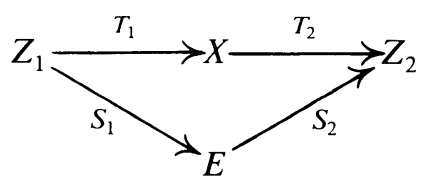

such that $S_{i}(i=1,2)$ is positive if $T_{i}$ is positive.

Moreover, if another scheme of operators

$$
Z_{1} \stackrel{P_{1}}{\rightarrow} X \stackrel{P_{2}}{\rightarrow} Z_{2}
$$

satisfies $0 \leqslant P_{i} \leqslant T_{i}(i=1,2)$, then there exists a factorization of $P_{2} P_{1}$ through the reflexive Banach lattice $E$

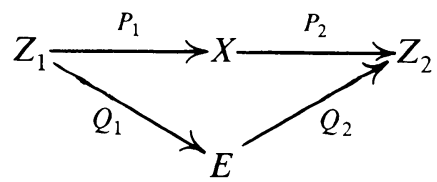

such that $0 \leqslant Q_{i} \leqslant S_{i}$ holds for each $i$. 
Proof. Consider two weakly compact operators $Z_{1} \stackrel{T_{1}}{\rightarrow} X \stackrel{T_{2}}{\rightarrow} Z_{2}$ between Banach spaces with $X$ a Banach lattice. Let $W$ be the convex solid hull of the relatively weakly compact set $T_{1}\left(B_{Z_{1}}\right)$ and consider the Banach lattice $(Y,\||\||)$ of Theorems 1.5 and 1.7. By Theorem 2.1 the Banach lattice $Y^{\prime}$ has order continuous norm.

Now if $S: Z_{1} \rightarrow Y$ is defined by $S(z)=T_{1}(z)$, then $S$ is continuous. On the other hand, if $T$ denotes the restriction of $T_{2}$ to $Y$, then (since every norm bounded subset of $Y$ is also norm bounded in $X$ ) the operator $T: Y \rightarrow Z_{2}$ is weakly compact. Clearly, the operators $Z_{1} \stackrel{S}{\rightarrow} Y \stackrel{T}{\rightarrow} Z_{2}$ satisfy $T_{2} T_{1}=T S$.

If $0 \leqslant P_{i} \leqslant T_{i}(i=1,2)$ holds, then clearly $P_{1}\left(Z_{1}\right) \subseteq Y$, and if $Z_{1} \stackrel{R_{1}}{\rightarrow} Y \stackrel{R_{2}}{\rightarrow} Z_{2}$ are defined by $R_{1}(z)=P_{1}(z)$ and $R_{2}(z)=P_{2}(z)$, then $0 \leqslant R_{1} \leqslant S, 0 \leqslant R_{2} \leqslant T$, and $P_{2} P_{1}=R_{2} R_{1}$ hold.

By Theorem 2.5 there exists a factorization of $T$ through a reflexive Banach lattice E

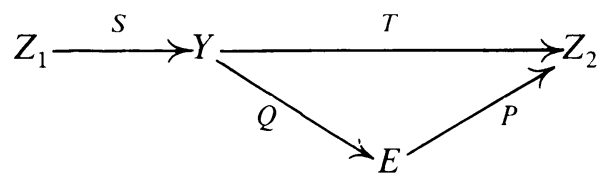

such that $Q$ is positive and with $P$ positive if $T$ is positive. Moreover, in case $0 \leqslant P_{2} \leqslant T_{2}$ holds, there exists an operator $Q_{2}: E \rightarrow Z_{2}$ satisfying $0 \leqslant Q_{2} \leqslant P$ and $R_{2}=Q_{2} Q$. To finish the proof, take $S_{1}=Q S, S_{2}=P$, and $Q_{1}=Q R_{1}$.

An important special case of Theorem 2.6 is the following

COROllaRy 2.7. If $T: X \rightarrow X$ is a weakly compact operator on a Banach lattice, then $T^{2}$ factors (with positive factors if $T$ is positive) through a reflexive Banach lattice.

Note. After submitting this paper, we learnt of the work of N. Ghoussoub and W. B. Johnson [12] which has some overlapping with ours.

We close this section with two problems related to the question in the abstract.

Problem 1. Does a weakly compact operator between two Banach lattices factor through a reflexive Banach lattice?

Problem 2. Does a positive weakly compact operator between two Banach lattices factor (if possible, with positive factors) through a reflexive Banach lattice?

3. Factoring compact operators. In this section we shall study when a compact operator has a factorization with compact factors through a reflexive Banach lattice. Some results of this type were obtained in [6].

We start the section with the "compact operator" analogue of Theorem 2.5.

THEOREM 3.1. Let $T: X \rightarrow Z$ be a compact operator from a Banach lattice $X$ into a Banach lattice space $Z$. If $X^{\prime}$ has order continuous norm, then $T$ admits a factorization

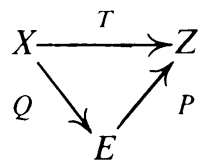


through a reflexive Banach lattice $E$ with the factor $Q$ a lattice homomorphism and the factor $P$ compact. In case $T$ is also positive, then $P$ can be chosen to be a positive operator.

Moreover, if another operator $S: X \rightarrow Z$ satisfies $0 \leqslant S \leqslant T$, then $S$ admits a factorization through the reflexive Banach lattice $E$

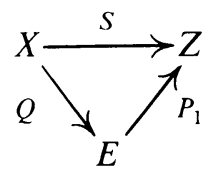

with $0 \leqslant P_{1} \leqslant P$.

Proof. Repeat the proof of Theorem 2.5, and use Lemma 1.6 to see that $R$ : $Z^{\prime} \rightarrow Y$ is a compact operator which implies that $P$ is a compact operator.

The next theorem extends Theorem 2.6 and is the major factorization result of the paper.

THEOREM 3.2. Consider the scheme of operators

$$
Z_{1} \stackrel{T_{1}}{\rightarrow} X \stackrel{T_{2}}{\rightarrow} Z_{2}
$$

between Banach spaces with each $T_{i}(i=1,2)$ compact or weakly compact. If $X$ is a Banach lattice, then there exists a reflexive Banach lattice $E$ and a factorization of $T_{2} T_{1}$

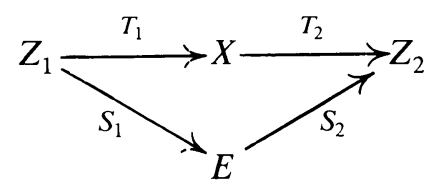

such that $S_{i}(i=1,2)$ has the same compactness property as $T_{i}$ and is positive if $T_{i}$ is positive.

Moreover, if another scheme of operators

$$
Z_{1} \stackrel{P_{1}}{\rightarrow} X \stackrel{P_{2}}{\rightarrow} Z_{2}
$$

satisfies $0 \leqslant P_{i} \leqslant T_{i}(i=1,2)$, then there exists a factorization of $P_{2} P_{1}$ through the reflexive Banach lattice $E$

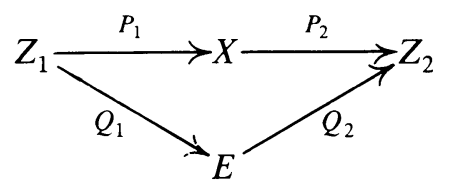

such that $0 \leqslant Q_{i} \leqslant S_{i}$ holds for each $i$.

Proof. Assume that $T_{1}$ is compact or weakly compact, and let $W$ denote the convex solid hull of the relatively weakly compact set $T_{1}\left(B_{Z_{1}}\right)$. If $Y$ denotes the Banach lattice determined by $W$, then (by Theorem 2.1) $Y^{\prime}$ has order continuous 
norm, and $T_{1}$ admits the factorization

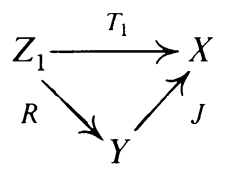

where $R(z)=T_{1}(z)$ for all $z \in Z_{1}$. Note that $J$ is a positive operator and that $R$ is positive if $T_{1}$ is positive.

The proof will be based upon the following diagram:

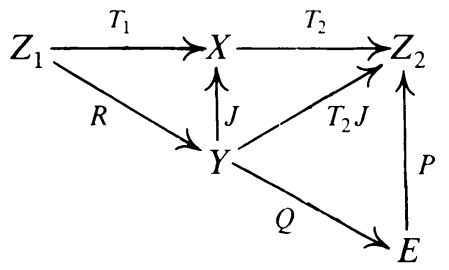

The factorization $T_{2} J=P Q$ will be through an appropriate reflexive Banach lattice $E$ that will be explained below. In all cases the desired factors will be $S_{1}=Q R$ and $S_{2}=P$. As we shall see, $Q$ always will be a positive operator, and $P$ will be positive if $T_{2}$ is positive.

Case $1 . T_{1}$ and $T_{2}$ are both weakly compact.

The factorization of $T_{2} J$ through a reflexive Banach lattice $E$ follows from Theorem 2.5.

Case 2. $T_{1}$ is weakly compact and $T_{2}$ is compact. By Theorem 3.1 the compact operator $T_{2} J$ factors through a reflexive Banach lattice $E$ with $P$ compact.

Case 3. $T_{1}$ is compact and $T_{2}$ is weakly compact. By Lemma 1.6 the operator $R$ is compact, and by Theorem 2.5 the weakly compact operator $T_{2} J$ factors through a reflexive Banach lattice $E$.

Case 4. $T_{1}$ and $T_{2}$ are both compact. As above, $R$ is compact, and by Theorem 3.1 the compact operator $T_{2} J$ factors through a reflexive Banach lattice $E$ with $P$ compact. Therefore, $S_{1}$ and $S_{2}$ are both compact.

Finally, note that $S_{i}$ is positive if $T_{i}$ is positive, and the rest of the proof follows from Theorem 2.6.

An immediate consequence of the preceding theorem is the following.

COROllary 3.3. If $T: X \rightarrow X$ is a compact operator on a Banach lattice, then $T^{2}$ factors with compact factors through a reflexive Banach lattice.

If $T$ is also positive, then the factors can be taken to be positive compact operators.

It was shown in [9] that if $E^{\prime}$ and $F$ have order continuous norms, then every positive compact operator from $E$ into $F$ dominated by a compact operator is itself compact. (Recall that an operator $S$ is dominated by another operator $T$ whenever $S \leqslant T$ holds.) Next, we shall present a proof of this result using factorization.

THEOREM 3.4 (DodDS - Fremlin). Let $E$ and $F$ be two Banach lattices such that $E^{\prime}$ and $F$ have order continuous norms. If a positive operator $T: E \rightarrow F$ is dominated by a compact operator, then $T$ is a compact operator. 
Proof. By Theorem 3.1 there exists a reflexive Banach lattice $G$ and a factorization of $T$

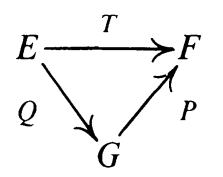

such that $P$ is positive dominated by a compact operator. By [5, Theorem 2.6, p. 234], $P$ is a Dunford-Pettis operator, and hence, a compact operator. Thus, $T$ is a compact operator.

The next result generalizes [3, Theorem 2.5, p. 296].

THEOREM 3.5. Consider the scheme of positive operators

$$
E \stackrel{T_{1}}{\rightarrow} X \stackrel{T_{2}}{\rightarrow} F
$$

between Banach lattices. If $F$ has order continuous norm, $T_{1}$ is dominated by a weakly compact operator, and $T_{2}$ is dominated by a compact operator, then $T_{2} T_{1}$ is a compact operator.

Dually, if $E^{\prime}$ has order continuous norm, $T_{1}$ is dominated by a compact operator, and $T_{2}$ is dominated by a weakly compact operator, then $T_{2} T_{1}$ is a compact operator.

Proof. By Theorem 3.2 there exist a reflexive Banach lattice $G$ and a factorization of $T_{2} T_{1}$

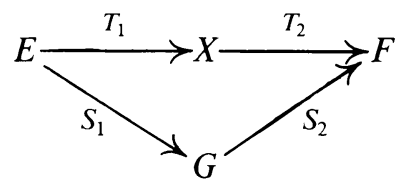

with $S_{2}$ positive dominated by a compact operator. By Theorem $3.4, S_{2}$ is compact, and hence, $T_{2} T_{1}$ is also compact.

The following theorem generalizes [3, Theorem 2.4 , p. 296].

THEOREM 3.6. Consider the scheme of positive operators

$$
E \stackrel{F_{1}}{\rightarrow} X \stackrel{T_{2}}{\rightarrow} Z \stackrel{T_{3}}{\rightarrow} F
$$

between Banach lattices such that $T_{1}$ is dominated by a weakly compact operator and $T_{2}$ by a compact operator. If $T_{3}$ is dominated either by a weakly compact or by $a$ Dunford-Pettis operator, then $T_{3} T_{2} T_{1}$ is a compact operator.

Proof. The proof is based upon the following diagram:

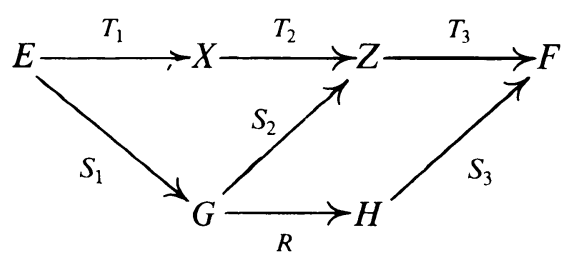


According to Theorem 3.2 the scheme $E \stackrel{T_{1}}{\rightarrow} X \stackrel{T_{2}}{\rightarrow} Z$ factors through a reflexive Banach lattice $G$ with $S_{2}$ positive dominated by a compact operator.

Assume first that $T_{3}$ is dominated by a Dunford-Pettis operator. Then by [5, Theorem 3.3, p. 236] the operator $T_{3} S_{2}$ is Dunford-Pettis, and so, $T_{3} T_{2} T_{1}=\left(T_{3} S_{2}\right) S_{1}$ is a compact operator.

Now assume that $T_{3}$ is dominated by a weakly compact operator. Then by Theorem 3.2 the scheme $G \stackrel{S_{2}}{\rightarrow} Z \stackrel{T_{3}}{\rightarrow} F$ factors through a reflexive Banach lattice $H$ with the factor $R$ positive dominated by a compact operator. By Theorem $3.4, R$ is compact, and so, $T_{3} T_{2} T_{1}=S_{3} R S_{1}$ is also compact.

In the preceding theorem the case when $T_{1}$ and $T_{3}$ were dominated by weakly compact operators was established in [13] by a different method.

Finally, it should be mentioned that W. B. Johnson has pointed out to us that his techniques in [14] yield also the following result: If $T: Z \rightarrow X$ is a compact operator and $X$ is a Banach lattice with the approximation property, then $T$ factors with compact factors (which can be taken positive if $T$ is positive) through a reflexive Banach space with an unconditional basis.

We close the paper with the corresponding open problems for compact operators.

Problem 3. Does a compact operator between two Banach lattices factor with compact factors through a reflexive Banach lattice?

Problem 4. Does a positive compact operator between two Banach lattices factor with compact factors (if possible, with positive compact factors) through a reflexive Banach lattice?

\section{REFERENCES}

1. Ju. A. Abramovič, Weakly compact sets in topological $K$-spaces, Teor. Funktsii. Funktsional. Anal. i Prilozhen 15 (1972), 27-35. (Russian) MR 46 \# 5982.

2. C. D. Aliprantis and O. Burkinshaw, Locally solid Riesz spaces, Academic Press, New York and London, 1978.

3. Positive compact operators on Banach lattices, Math. Z. 174 (1980), 289-298.

4. On weakly compact operators on Banach lattices, Proc. Amer. Math. Soc. 83 (1981), $573-578$.

5. _ Dunford-Pettis operators on Banach lattices, Trans. Amer. Math. Soc. 274 (1982), 227-238.

6. A. B. Buhvalov, Factorization of compact operators and an example of a reflexive Banach lattice without the approximation property, Soviet Math. Dokl. 17 (1976), 423-426.

7. W. J. Davis, T. Figiel, W. B. Johnson and A. Pelczynski, Factoring weakly compact operators, J. Funct. Anal. 17 (1974), 311-327.

8. J. J. Diestel and J. J. Uhl, Jr., Vector measures, Math. Surveys, No. 15, Amer. Math. Soc., Providence, R. I., 1977.

9. P. G. Dodds and D. H. Fremlin, Compact operators in Banach lattices, Israel J. Math. 34 (1979), 287-320.

10. T. Figiel, Factorization of compact operators and applications to the approximation property, Studia Math. 45 (1973), 191-210.

11. T. Figiel, W. B. Johnson and L. Tzafriri, On Banach lattices and spaces having local unconditional structure, with applications to Lorentz function spaces, J. Approximation Theory 13 (1975), 395-412.

12. N. Ghoussoub and W. B. Johnson, On subspaces of Banach lattices not containing $C(\Delta)$ (to appear).

13. W. Haid, Sätze vom Radon-Nikodym-typ für operatoren auf Banachverbänden, Ph. D. Dissertation, University of Tübingen, 1982.

14. W. B. Johnson, Factoring compact operators, Israel J. Math. 9 (1971), 337-345. 
15. H. P. Lotz, Extensions and liftings of positive linear mappings on Banach lattices, Trans. Amer. Math. Soc. 211 (1975), 85-100.

16. P. Meyer-Nieberg, Zur schwachen kompaktheit in Banachverbänden, Math. Z. 134 (1973), 303-315.

17. H. H. Schaefer, Banach lattices and positive operators, Springer-Verlag, Heidelberg, Berlin and New York, 1974.

18. A. W. Wickstead, Extremal structure of cones of operators, Quart. J. Math. Oxford Ser. (2) 32 (1981), 239-253.

Department of Mathematical Sciences, Indiana University - Purdue University at IndianapOLIS, INDIANAPOLIS, INDIANA 46223 